\title{
LEGENDRE COLLOCATION METHOD FOR SOLVING NONLINEAR DIFFERENTIAL EQUATIONS
}

\author{
Aysun Güner and Salih Yalçınbaş \\ Department of Mathematics, Celal Bayar University, 45140, Muradiye, Manisa, Turkey \\ aysun-guner-20@hotmail.com salih.yalcinbas@cbu.edu.tr
}

\begin{abstract}
In this study, a matrix method based on Legendre collocation points on interval $[-1,1]$ is proposed for the approximate solution of the some first order nonlinear ordinary differential equations with the mixed conditions in terms of Legendre polynomials. The method by means of Legendre collocation points, transforms the differential equation to a matrix equation which corresponds to a system of nonlinear algebraic equations with unknown Legendre coefficients. Also, the method can be used for solving Riccati equation. The numerical results show the effectuality of the method for this type of equations. Comparisons are made between the obtained solution and the exact solution.
\end{abstract}

Key Words- Nonlinear ordinary differential equations, Legendre polynomials and series, Legendre collocation points, Legendre collocation method.

\section{INTRODUCTION}

Nonlinear ordinary differential equations are frequently used to model a wide class of problems in many areas of scientific fields; chemical reactions, spring-mass systems bending of beams, resistor-capacitor-inductance circuits, pendulums, the motion of a rotating mass around another body and so forth [1,2]. These equations have also demonstrated their usefulness in ecology, economics, biology, astrophysics and engineering. Thus, methods of solution for these equations are of great importance to engineers and scientists. However many important differential equations can be solved by well known analytical techniques, a greater number of physically significant differential equations can not be solved $[1,3,4]$.

Moreover, nonlinear differential equations play afundamental role in control theory; for example, optimal control, filtering and estimation and order reduction, etc. $[5,6]$.

We consider the approximate solution of the first order nonlinear ordinary differential equation

$$
\begin{aligned}
& N\left(x_{i}\right)\left(y^{\prime}\left(x_{i}\right)\right)^{2}+Q\left(x_{i}\right) y\left(x_{i}\right) y^{\prime}\left(x_{i}\right)+R\left(x_{i}\right) y^{\prime}\left(x_{i}\right)+S\left(x_{i}\right) y\left(x_{i}\right)+T\left(x_{i}\right) y^{2}\left(x_{i}\right)=g\left(x_{i}\right) \\
& a \leq x \leq b \\
& \text { under the mixed condition } \\
& \quad \alpha y(a)+\beta y(b)=\lambda
\end{aligned}
$$

where $N(x), Q(x), R(x), S(x), T(x)$ and $g(x)$ are the functions defined on $a \leq x \leq b$; the real coefficients $\alpha, \beta$ and $\lambda$ are appropriate constants. 
Our purpose is to obtain an approximate solution of (1) in the following Legendre polynomial form

$$
y(x)=\sum_{n=0}^{N} a_{n} P_{n}(x), \quad-1 \leq x \leq 1
$$

where $a_{n},(n=0,1,2, \ldots, N)$ are unknown Legendre coefficients.

Here $P_{n}(x), n=0,1,2 \ldots N$ are the Legendre polynomials defined by

$$
\begin{aligned}
\mathbf{P}_{n}(x) & =\frac{1}{2^{n}} \sum_{k=0}^{\left[\frac{n}{2}\right]}(-1)^{k}\left(\begin{array}{l}
n \\
k
\end{array}\right)\left(\begin{array}{c}
2 n-2 k \\
n
\end{array}\right) x^{n-2 k}, n=0,1,2 \ldots \\
{\left[\frac{n}{2}\right] } & =\left\{\begin{array}{cc}
\frac{n}{2}, & n \text { even } \\
\frac{n-1}{2}, & n \text { odd }
\end{array}\right.
\end{aligned}
$$

\section{FUNDAMENTAL MATRIX RELATIONS}

Let us consider the nonlinear differential equation (1) and find the matrix forms of each term in the equation. Firstly, we consider the solution $y(x)$ defined by a truncated series (3) and then we can convert to the matrix form

where

$$
y(x)=\mathbf{P}(x) \mathbf{A}
$$

$$
\begin{aligned}
& \mathbf{P}(x)=\left[\begin{array}{lllll}
\mathbf{P}_{0}(x) & \mathbf{P}_{1}(x) & \ldots & \mathbf{P}_{N}(x)
\end{array}\right] \\
& \mathbf{A}=\left[\begin{array}{llll}
a_{0} & a_{1} & \ldots & a_{N}
\end{array}\right]^{T}
\end{aligned}
$$

Let us write the solution $y^{\prime}(x), y(x) y^{\prime}(x)$ and $\left(y^{\prime}(x)\right)^{2}$ in the matrix form. Firstly, if we differentiate expression (4) with respect to $x$, we obtain

$$
\begin{aligned}
y^{\prime}(x) & =\mathbf{P}^{\prime}(\mathbf{x}) \mathbf{A} \\
& =\mathbf{P}(x) \boldsymbol{\Pi}^{\mathbf{T}} \mathbf{A}
\end{aligned}
$$

where if $n$ is even

$$
\boldsymbol{\Pi}=\left[\begin{array}{cccccccc}
0 & 0 & 0 & 0 & \cdots & 0 & 0 & 0 \\
1 & 0 & 0 & 0 & \cdots & 0 & 0 & 0 \\
0 & 3 & 0 & 0 & \cdots & 0 & 0 & 0 \\
1 & 0 & 5 & 0 & \cdots & 0 & 0 & 0 \\
\vdots & \vdots & \vdots & \vdots & \vdots & \vdots & \vdots & \vdots \\
0 & 3 & 0 & 7 & \cdots & 2 N-3 & 0 & 0 \\
1 & 0 & 5 & 0 & \cdots & 0 & 2 N-1 & 0
\end{array}\right]_{(N+1) \times(N+1)}
$$


if $n$ is odd

$$
\boldsymbol{\Pi}=\left[\begin{array}{cccccccc}
0 & 0 & 0 & 0 & \cdots & 0 & 0 & 0 \\
1 & 0 & 0 & 0 & \cdots & 0 & 0 & 0 \\
0 & 3 & 0 & 0 & \cdots & 0 & 0 & 0 \\
1 & 0 & 5 & 0 & \cdots & 0 & 0 & 0 \\
\vdots & \vdots & \vdots & \vdots & \vdots & \vdots & \vdots & \vdots \\
1 & 0 & 5 & 0 & \cdots & 2 N-3 & 0 & 0 \\
0 & 3 & 0 & 7 & \cdots & 0 & 2 N-1 & 0
\end{array}\right]_{(N+1) \times(N+1)}
$$

On the other hand, the matrix form of expression $y^{2}(x)$ is obtained as

$$
y^{2}(x)=\left[\begin{array}{llllll}
1 & x & \frac{1}{2}\left(3 x^{2}-1\right) & \cdots & \cdots
\end{array}\right]\left[\begin{array}{cccc}
\mathbf{P}(\mathrm{x}) & 0 & \cdots & 0 \\
0 & \mathbf{P}(\mathrm{x}) & \cdots & 0 \\
\vdots & \vdots & \ddots & \vdots \\
0 & 0 & \cdots & \mathbf{P}(\mathrm{x})
\end{array}\right]\left[\begin{array}{c}
\mathrm{a}_{0} \mathbf{A} \\
\mathrm{a}_{1} \mathbf{A} \\
\vdots \\
\mathrm{a}_{\mathrm{N}} \mathbf{A}
\end{array}\right]
$$

or shortly

$$
y^{2}(x)=\mathbf{P}(x) \mathbf{P}^{*}(x) \mathbf{A}^{*}
$$

where

$$
\begin{aligned}
& \mathbf{P}^{*}(\mathrm{x})=\left[\begin{array}{cccc}
\mathbf{P}(\mathrm{x}) & 0 & \cdots & 0 \\
0 & \mathbf{P}(\mathrm{x}) & \cdots & 0 \\
\vdots & \vdots & \ddots & \vdots \\
0 & 0 & \cdots & \mathbf{P}(\mathrm{x})
\end{array}\right] \\
& \mathbf{A}^{*}=\left[\begin{array}{llll}
a_{0} \mathbf{A} & a_{1} \mathbf{A} & \cdots & a_{N} \mathbf{A}
\end{array}\right]^{T} \\
& =\left[\begin{array}{llllllllllll}
a_{0} a_{0} & a_{0} a_{1} & \cdots & a_{0} a_{N} & a_{1} a_{1} & \cdots & a_{1} a_{N} & \cdots & a_{N} a_{0} & a_{N} a_{1} & \cdots & a_{N} a_{N}
\end{array}\right]
\end{aligned}
$$

By using the expression (4), (5) and (6) we obtain

$$
y(x) y^{\prime}(x)=\mathbf{P}(x) \mathbf{P}^{*}(x)\left(\boldsymbol{\Pi}^{\mathbf{T}}\right)^{*} \mathbf{A}^{*}
$$

Following a similar way to (6), we have

$$
\left(y^{\prime}(x)\right)^{2}=\mathbf{P}(x) \boldsymbol{\Pi}^{\mathbf{T}} \mathbf{P}^{*}(x)\left(\boldsymbol{\Pi}^{\mathbf{T}}\right)^{*} \mathbf{A}^{*}
$$

where 


$$
\left(\boldsymbol{\Pi}^{\mathbf{T}}\right)^{*}(\mathbf{x})=\left[\begin{array}{cccc}
\boldsymbol{\Pi}^{\mathbf{T}} & 0 & \cdots & 0 \\
0 & \boldsymbol{\Pi}^{\mathbf{T}} & \cdots & 0 \\
\vdots & \vdots & \ddots & \vdots \\
0 & 0 & 0 & \boldsymbol{\Pi}^{\mathbf{T}}
\end{array}\right]
$$

\section{MATRIX RELATIONS BASED ON COLLOCATION POINTS}

Let us use the collocation points defined by

$$
x_{i}=a+\frac{b-a}{N} i, \quad i=0,1,2, \ldots, N
$$

in order to

$$
a=x_{0}<x_{1}<\cdots<x_{n}=b
$$

By using the collocation points (9) into Eq. (1), we obtain the equation

$$
\begin{aligned}
& N\left(x_{i}\right)\left(y^{\prime}\left(x_{i}\right)\right)^{2}+Q\left(x_{i}\right) y\left(x_{i}\right) y^{\prime}\left(x_{i}\right)+R\left(x_{i}\right) y^{\prime}\left(x_{i}\right)+S\left(x_{i}\right) y\left(x_{i}\right)+T\left(x_{i}\right) y^{2}\left(x_{i}\right)=g\left(x_{i}\right) \\
& i=0,1,2, \ldots, N ; a \leq x \leq b
\end{aligned}
$$

By using the relations (4), (5), (6), (7) and (8); the system (10) can be written in the matrix form or briefly

$$
\left(\mathbf{N P} \boldsymbol{\Pi}^{\mathbf{T}} \mathbf{P}^{*}\left(\boldsymbol{\Pi}^{\mathbf{T}}\right)^{*}+\mathbf{Q P} \mathbf{P}^{*}\left(\boldsymbol{\Pi}^{\mathbf{T}}\right)^{*}+\mathbf{T} \mathbf{P} \mathbf{P}^{*}\right) \mathbf{A}^{*}+\left(\mathbf{R P} \boldsymbol{\Pi}^{\mathbf{T}}+\mathbf{S P}\right) \mathbf{A}=\mathbf{G}
$$

$$
\mathbf{W A}^{*}+\mathbf{V A}=\mathbf{G}
$$

where

$$
\begin{aligned}
& \mathbf{N}=\left[\begin{array}{cccc}
N\left(\mathrm{x}_{0}\right) & 0 & \cdots & 0 \\
0 & N\left(\mathrm{x}_{1}\right) & \cdots & 0 \\
\vdots & \vdots & \ddots & \vdots \\
0 & 0 & \cdots & N\left(\mathrm{x}_{N}\right)
\end{array}\right] ; \quad \mathbf{Q}=\left[\begin{array}{cccc}
Q\left(\mathrm{x}_{0}\right) & 0 & \cdots & 0 \\
0 & Q\left(\mathrm{x}_{1}\right) & \cdots & 0 \\
\vdots & \vdots & \ddots & \vdots \\
0 & 0 & \cdots & Q\left(\mathrm{x}_{N}\right)
\end{array}\right] \\
& \mathbf{R}=\left[\begin{array}{cccc}
R\left(\mathrm{x}_{0}\right) & 0 & \cdots & 0 \\
0 & R\left(\mathrm{x}_{1}\right) & \cdots & 0 \\
\vdots & \vdots & \ddots & \vdots \\
0 & 0 & \cdots & R\left(\mathrm{x}_{N}\right)
\end{array}\right] \quad ; \quad \mathbf{S}=\left[\begin{array}{cccc}
S\left(\mathrm{x}_{0}\right) & 0 & \cdots & 0 \\
0 & S\left(\mathrm{x}_{1}\right) & \cdots & 0 \\
\vdots & \vdots & \ddots & \vdots \\
0 & 0 & \cdots & S\left(\mathrm{x}_{N}\right)
\end{array}\right] \\
& \mathbf{T}=\left[\begin{array}{cccc}
T\left(\mathrm{x}_{0}\right) & 0 & \cdots & 0 \\
0 & T\left(\mathrm{x}_{1}\right) & \cdots & 0 \\
\vdots & \vdots & \ddots & \vdots \\
0 & 0 & \cdots & T\left(\mathrm{x}_{N}\right)
\end{array}\right] \quad ; \quad \mathbf{G}=\left[\begin{array}{cc}
g\left(x_{0}\right) \\
g\left(x_{1}\right) \\
\vdots \\
g\left(x_{N}\right)
\end{array}\right]
\end{aligned}
$$




$$
\begin{gathered}
\mathbf{P}=\left[\begin{array}{c}
\mathbf{P}\left(x_{0}\right) \\
\mathbf{P}\left(x_{1}\right) \\
\vdots \\
\mathbf{P}\left(x_{N}\right)
\end{array}\right]=\left[\begin{array}{ccccc}
\mathrm{P}_{0}\left(x_{0}\right) & \mathrm{P}_{1}\left(x_{0}\right) & \mathrm{P}_{2}\left(x_{0}\right) & \cdots & \mathrm{P}_{N}\left(x_{0}\right) \\
\mathrm{P}_{0}\left(x_{1}\right) & \mathrm{P}_{1}\left(x_{1}\right) & \mathrm{P}_{2}\left(x_{1}\right) & \cdots & \mathrm{P}_{N}\left(x_{1}\right) \\
\vdots & \vdots & \vdots & \ddots & \vdots \\
\mathrm{P}_{0}\left(x_{N}\right) & \mathrm{P}_{1}\left(x_{N}\right) & \mathrm{P}_{2}\left(x_{N}\right) & \cdots & \mathrm{P}_{N}\left(x_{N}\right)
\end{array}\right] \\
\mathbf{P}^{*}(\mathrm{x})=\left[\begin{array}{cccc}
\mathbf{P}\left(\mathrm{x}_{0}\right) & 0 & \cdots & 0 \\
0 & \mathbf{P}\left(\mathrm{x}_{1}\right) & \cdots & 0 \\
\vdots & \vdots & \ddots & \vdots \\
0 & 0 & \cdots & \mathbf{P}\left(\mathrm{x}_{\mathrm{N}}\right)
\end{array}\right]
\end{gathered}
$$

\section{METHOD OF SOLUTION}

Therefore, the fundamental matrix equation (11) corresponding to Eq. (1) can be written in the augmented form

$\mathbf{W A}^{*}+\mathbf{V A}=\mathbf{G}$

or

$$
[\mathbf{W} ; \mathbf{V} ; \mathbf{G}]
$$

where

$$
\begin{aligned}
& \mathbf{W}=\left[w_{p q}\right]=\mathbf{N} \mathbf{P} \boldsymbol{\Pi}^{\mathbf{T}} \mathbf{P}^{*}\left(\boldsymbol{\Pi}^{\mathbf{T}}\right)^{*}+\mathbf{Q P} \mathbf{P}^{*}\left(\boldsymbol{\Pi}^{\mathbf{T}}\right)^{*}+\mathbf{T} \mathbf{P} \mathbf{P}^{*} ; \quad \mathbf{p}, \mathbf{q}=0,1,2, \ldots \mathbf{N} \\
& \mathbf{V}=\left[v_{p q}\right]=\mathbf{R} \mathbf{P} \boldsymbol{\Pi}^{\mathbf{T}}+\mathbf{S P} .
\end{aligned}
$$

We can find the corresponding matrix equation for the condition (2), using the relation (4), as follows:

$$
\{\alpha \mathbf{P}(a)+\beta \mathbf{P}(\mathrm{b})\} \mathbf{A}=[\lambda]
$$

so that

$$
\begin{aligned}
& \mathbf{P}(a)=\left[\begin{array}{llll}
1 & a & \frac{1}{2}\left(3 a^{2}-1\right) & \cdots
\end{array}\right] \\
& \mathbf{P}(b)=\left[\begin{array}{llll}
1 & b & \frac{1}{2}\left(3 b^{2}-1\right) & \cdots
\end{array}\right]
\end{aligned}
$$

We can write the corresponding matrix form (13) for the mixed condition (2) in the augmented matrix form as

$$
[\mathbf{Z} ; \mathbf{0}: \lambda]
$$

where

$$
\mathbf{Z}=\left[\begin{array}{llll}
z_{0} & z_{1} & \cdots & z_{N}
\end{array}\right]=\alpha \mathbf{P}(\mathrm{a})+\beta \mathbf{P}(\mathrm{b})
$$




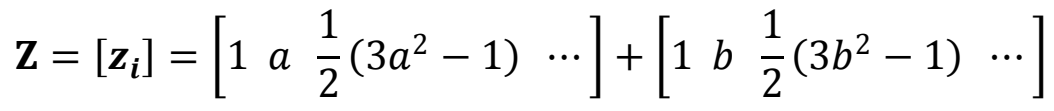

$$
\begin{aligned}
& \mathbf{0}=\left[\begin{array}{llll}
0 & 0 & \cdots & 0
\end{array}\right]_{1 \times(N+1)} .
\end{aligned}
$$

To obtain the approximate solution of Eq. (1) with the mixed condition (2) in the terms of Legendre polynomials, by replacing the row matrix (14) by the last row of the matrix (11), we obtain the required augmented matrix:

$[\overline{\mathbf{W}} ; \overline{\mathbf{V}} ; \overline{\mathbf{G}}]=\left[\begin{array}{ccccccccccccc}w_{00} & w_{01} & w_{02} & \cdots & w_{0 N} & ; & v_{00} & v_{01} & v_{02} & \cdots & v_{0 N} & : & g\left(x_{0}\right) \\ w_{10} & w_{11} & w_{12} & \cdots & w_{1 N} & ; & v_{10} & v_{11} & v_{12} & \cdots & v_{1 N} & \vdots & g\left(x_{1}\right) \\ \vdots & \vdots & \vdots & \ddots & \vdots & ; & \vdots & \vdots & \vdots & \ddots & \vdots & \vdots & \vdots \\ w_{N-1,0} & w_{N-1,1} & w_{N-1,2} & \cdots & w_{N-1, N} & ; & v_{N-1,0} & v_{N-1,1} & v_{N-1,2} & \cdots & v_{N-1, N} & \vdots & g\left(x_{N}\right) \\ 0 & 0 & 0 & \cdots & 0 & ; & z_{0} & z_{1} & z_{2} & \cdots & z_{N} & \vdots & \lambda\end{array}\right]$

or the corresponding matrix equation

$\overline{\mathbf{W}} \mathbf{A}^{*}+\overline{\mathbf{V}} \mathbf{A}=\overline{\boldsymbol{G}}$

where

$$
\overline{\mathbf{W}}=\left[\begin{array}{cccc}
\mathrm{w}_{00} & \mathrm{w}_{01} & \cdots & \mathrm{w}_{0 \mathrm{~N}} \\
\mathrm{w}_{10} & \mathrm{w}_{11} & \cdots & \mathrm{w}_{1 \mathrm{~N}} \\
\vdots & \vdots & \ddots & \vdots \\
\mathrm{w}_{\mathrm{N}-1,0} & \mathrm{w}_{\mathrm{N}-1,1} & \cdots & \mathrm{w}_{\mathrm{N}-1, \mathrm{~N}} \\
0 & 0 & \cdots & 0
\end{array}\right] \overline{\mathbf{V}}=\left[\begin{array}{cccc}
\mathrm{v}_{00} & \mathrm{v}_{01} & \cdots & \mathrm{v}_{0 \mathrm{~N}} \\
\mathrm{v}_{10} & \mathrm{v}_{11} & \cdots & \mathrm{v}_{1 \mathrm{~N}} \\
\vdots & \vdots & \ddots & \vdots \\
\mathrm{v}_{\mathrm{N}-1,0} & \mathrm{v}_{\mathrm{N}-1,1} & \cdots & \mathrm{v}_{\mathrm{N}-1, \mathrm{~N}} \\
z_{0} & z_{1} & \cdots & z_{N}
\end{array}\right] \overline{\mathbf{G}}=\left[\begin{array}{c}
g\left(x_{0}\right) \\
g\left(x_{1}\right) \\
\vdots \\
g\left(x_{N-1}\right) \\
\lambda
\end{array}\right] .
$$

The unknown coefficients set $\left\{a_{0}, a_{1}, \ldots, a_{N}\right\}$ can be determined from the nonlinear system (15). As a result, we can obtain approximate solution in the truncated series form (3).

\section{ACCURACY OF SOLUTION}

We can check the accuracy of the method. The truncated Legendre series in (3) have to be approximately satisfying Eq. (1). For each $x=x_{i} \in[a, b], i=1,2, \ldots, N$ $E\left(x_{i}\right)=\left|N\left(x_{i}\right)\left(y^{\prime}\left(x_{i}\right)\right)^{2}+Q\left(x_{i}\right) y\left(x_{i}\right) y^{\prime}\left(x_{i}\right)+R\left(x_{i}\right) y^{\prime}\left(x_{i}\right)+S\left(x_{i}\right) y\left(x_{i}\right)+T\left(x_{i}\right) y^{2}\left(x_{i}\right)-g\left(x_{i}\right)\right| \cong 0$ and $E\left(x_{i}\right) \leq 10^{-k_{i}}$ ( $k_{i}$ is any positive integer). 
If $\max \left(10^{-k_{i}}\right)=10^{-k}$ ( $k$ is any positive integer) is prescribed, then the truncation limit $N$ is increased until the difference $E\left(x_{i}\right)$ at each of the points $x_{i}$ becomes smaller than the prescribed $10^{-k}[7-13]$.

\section{NUMERICAL EXAMPLES}

In this section, two numerical examples are given to show the accuracy and efficiency of the presented method.

Example 6.1. Let us first consider the first-order nonlinear differential equation

$$
\left(y^{\prime}(x)\right)^{2}-x^{2} y(x)=1-x^{3}
$$

with condition

$$
y(-1)+2 y(1)=1 \quad, \quad-1 \leq x \leq 1
$$

and the approximate solution $y(x)$ by the truncated Legendre polynomial

$$
y(x)=\sum_{n=0}^{2} a_{n} \mathrm{P}_{n}, \quad-1 \leq x \leq 1
$$

where

$$
\mathrm{N}(x)=1, \mathrm{Q}(x)=0, \mathrm{R}(x)=0, \mathrm{~S}(x)=-x^{2}, \mathrm{~T}(x)=0, \mathrm{~g}(x)=1-x^{3}
$$

For $N=2$ the collocation points become

$$
x_{0}=-1, x_{1}=0, x_{2}=1 \text {. }
$$

From the fundamental matrix equations for the given equation and condition respectively are obtained as

and

$$
\mathbf{N P} \Pi^{\mathrm{T}} \mathbf{P}^{*}\left(\Pi^{\mathrm{T}}\right)^{*} \mathbf{A}^{*}+\mathbf{S P A}=\mathbf{G}
$$

$$
\{\mathbf{P}(-1)+2 \mathbf{P}(1)\} \mathbf{A}=[1]
$$

so that

$$
\begin{aligned}
& \mathbf{N}=\left[\begin{array}{lll}
1 & 0 & 0 \\
0 & 1 & 0 \\
0 & 0 & 1
\end{array}\right], \quad \mathbf{A}=\left[\begin{array}{l}
a_{0} \\
a_{1} \\
a_{2}
\end{array}\right], \mathbf{G}=\left[\begin{array}{l}
2 \\
1 \\
0
\end{array}\right], \mathbf{P}=\left[\begin{array}{ccc}
1 & -1 & 1 \\
1 & 0 & -1 / 2 \\
1 & 1 & 1
\end{array}\right] \\
& \mathbf{Q}=\left[\begin{array}{lll}
0 & 0 & 0 \\
0 & 0 & 0 \\
0 & 0 & 0
\end{array}\right], \quad \mathbf{R}=\left[\begin{array}{lll}
0 & 0 & 0 \\
0 & 0 & 0 \\
0 & 0 & 0
\end{array}\right], \mathbf{S}=\left[\begin{array}{ccc}
-1 & 0 & 0 \\
0 & 0 & 0 \\
0 & 0 & -1
\end{array}\right], \mathbf{T}=\left[\begin{array}{lll}
0 & 0 & 0 \\
0 & 0 & 0 \\
0 & 0 & 0
\end{array}\right]
\end{aligned}
$$


$\mathbf{P}^{*}=\left[\begin{array}{ccccccccc}1 & -1 & 1 & 0 & 0 & 0 & 0 & 0 & 0 \\ 0 & 0 & 0 & 1 & 0 & -1 / 2 & 0 & 0 & 0 \\ 0 & 0 & 0 & 0 & 0 & 0 & 1 & 1 & 1\end{array}\right], \quad \Pi=\left[\begin{array}{lll}0 & 0 & 0 \\ 1 & 0 & 0 \\ 0 & 3 & 0\end{array}\right]$

$\mathbf{A}^{*}=\left[\begin{array}{lllllllll}a_{0} a_{0} & a_{0} a_{1} & a_{0} a_{2} & a_{1} a_{0} & a_{1} a_{1} & a_{1} a_{2} & a_{2} a_{0} & a_{2} a_{1} & a_{2} a_{2}\end{array}\right]^{T}$.

The augmented matrix for this fundamental matrix equation is calculated

$$
[\overline{\mathbf{W}} ; \overline{\mathbf{V}} ; \overline{\mathbf{G}}]=\left[\begin{array}{ccccccccccccccc}
0 & 0 & 0 & 0 & 1 & 0 & 0 & -3 & -9 & ; & -1 & 1 & -1 & : & 2 \\
0 & 0 & 0 & 0 & 1 & 0 & 0 & 0 & 0 & ; & 0 & 0 & 0 & : & 1 \\
0 & 0 & 0 & 0 & 0 & 0 & 0 & 0 & 0 & ; & 3 & 1 & 3 & : & 1
\end{array}\right]
$$

or

$$
\begin{aligned}
& a_{1}^{2}-3 a_{2} a_{1}-9 a_{2}{ }^{2}-a_{0}+a_{1}-a_{3}=2 \\
& a_{1}^{2}=1 \\
& 3 a_{0}+a_{1}+3 a_{2}=1
\end{aligned}
$$

From the obtained system, the coefficients $a_{0}, a_{1}$ and $a_{2}$ are found as

$$
a_{0}=0, a_{1}=1 \text { and } a_{2}=0
$$

Hence we have the Legendre polynomial solution

$$
y(x)=x
$$

Example 6.2. Consider the following nonlinear differential equation (Riccati Equation ) given by

$$
x y^{\prime}-x y+y^{2}=e^{2 x},-1 \leq x \leq 1
$$

with the initial condition

$$
y(0)=1
$$

So that

$$
\mathrm{N}(x)=0, Q(x)=0, R(x)=1, S(x)=-x, T(x)=1, g(x)=e^{2 x}
$$

The solutions obtained for $N=3,4,5$ are compared with the exact solution is $e^{x}$, which are given in Fig 1. We compare the numerical solution and absolute errors for $N=3,4,5$ in Table 1 . 
Table 1. Comparison of the absolute errors of Example 6.2

\section{Present method}

$N=3$

$\boldsymbol{x}_{\boldsymbol{i}}$ Exact Solution $\boldsymbol{y}\left(\boldsymbol{x}_{\boldsymbol{i}}\right)$

\begin{tabular}{llllllll}
\hline-1. & 0.367879 & 0.334323 & $3.355627 \mathrm{E}-02$ & 0.375008 & $7.129406 \mathrm{E}-03$ & 0.366674 & $1.205123 \mathrm{E}-03$ \\
-0.8 & 0.449329 & 0.435370 & $1.39587 \mathrm{E}-02$ & 0.451714 & $2.385336 \mathrm{E}-03$ & 0.449005 & $3.23411 \mathrm{E}-04$ \\
-0.6 & 0.548811 & 0.544421 & $4.390231 \mathrm{E}-03$ & 0.549368 & $5.565309 \mathrm{E}-04$ & 0.548751 & $5.977526 \mathrm{E}-05$ \\
-0.4 & 0.67032 & 0.669516 & $8.033632 \mathrm{E}-04$ & 0.670368 & $4.796019 \mathrm{E}-05$ & 0.670313 & $6.920744 \mathrm{E}-06$ \\
-0.2 & 0.818730 & 0.818696 & $3.456772 \mathrm{E}-05$ & 0.818712 & $1.854363 \mathrm{E}-05$ & 0.818729 & $1.547447 \mathrm{E}-06$ \\
0.0 & 1.000000 & 1.000000 & 0.0 & 1.000000 & 0.0 & 1.000000 & 0.0 \\
0.2 & 1.221403 & 1.221468 & $6.545648 \mathrm{E}-05$ & 1.221431 & $2.867506 \mathrm{E}-05$ & 1.221406 & $2.751472 \mathrm{E}-06$ \\
0.4 & 1.491825 & 1.491141 & $6.837805 \mathrm{E}-04$ & 1.491807 & $1.730121 \mathrm{E}-05$ & 1.491826 & $1.205291 \mathrm{E}-06$ \\
0.6 & 1.822119 & 1.817058 & $5.060605 \mathrm{E}-03$ & 1.82153 & $5.891915 \mathrm{E}-04$ & 1.822062 & $5.722084 \mathrm{E}-05$ \\
0.8 & 2.225541 & 2.20726 & $1.828079 \mathrm{E}-02$ & 2.222601 & $2.940306 \mathrm{E}-03$ & 2.225153 & $3.87694 \mathrm{E}-04$ \\
1.0 & 2.718282 & 2.669787 & $4.8495 \mathrm{E}-02$ & 2.708624 & $9.658009 \mathrm{E}-03$ & 2.716702 & $1.579906 \mathrm{E}-03$ \\
\hline
\end{tabular}

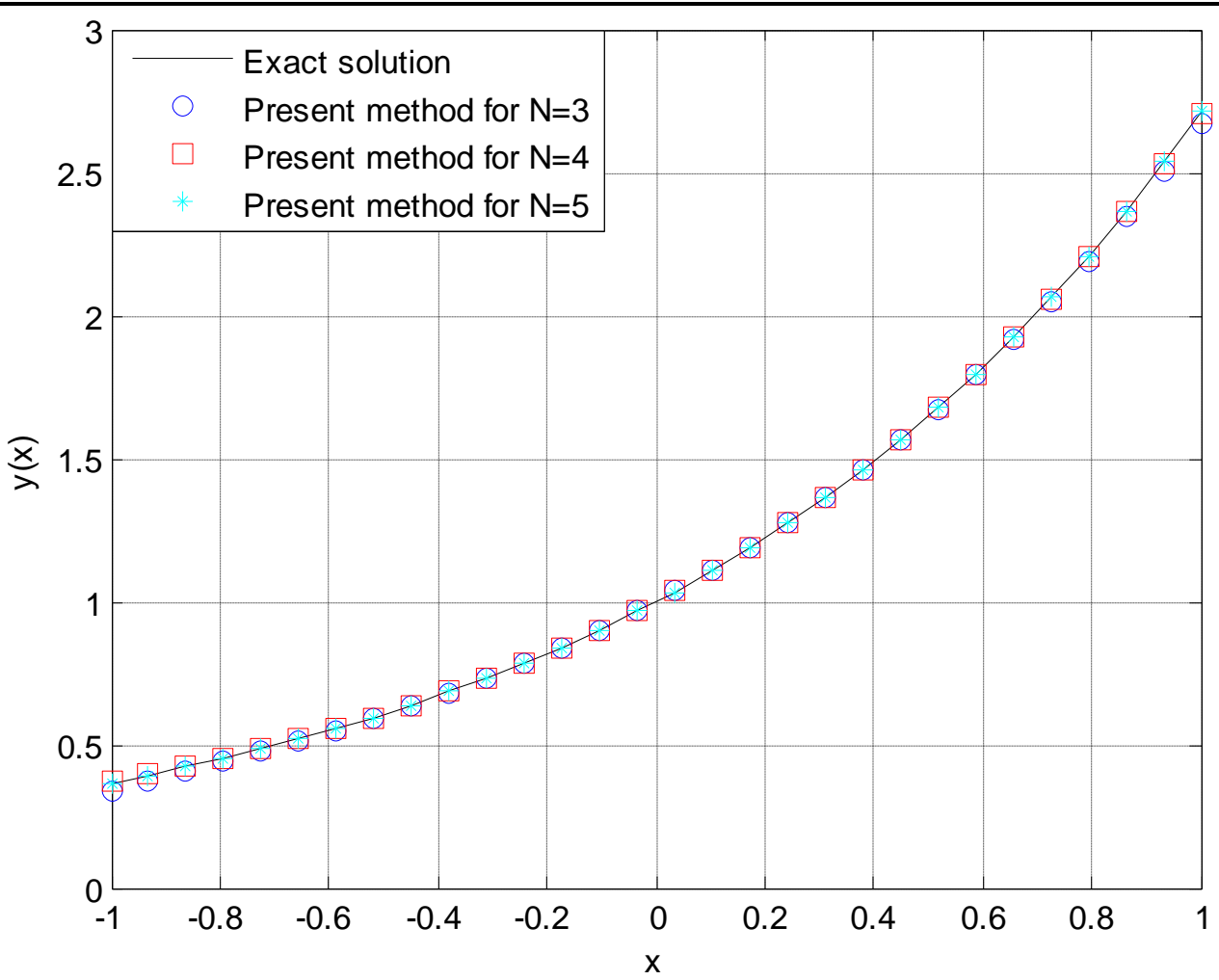

Figure 1. Numerical and exact solution of Example 6.2 for $\mathrm{N}=3,4,5$

$N=5$

Absolute Errors 
A Legendre collocation method for the approximate solutions of the some first order nonlinear ordinary differential equations on the interval $[-1,1]$ is analyzed in this study. A considerable advantage of the method is that the Legendre coefficients of the solution are found very easily by using computer programs. For this reason, this process is much faster than the other methods. Legendre collocation method gives well results for the different values $N$. The method can also be extended to the high order nonlinear ordinary differential equations with variable coefficients, but some modifications are required.

\section{REFERENCES}

1. H. Bulut, D. J. Evens, On the solution of Riccati equation by the decomposition method, International Journal of Computer Mathematics 79(1), 103-109, doi: 10.1080/00207160211917, 2002.

2. G. F. Corliss, Guarented Error Bounds for Ordinary Differential Equations, in Theory and Numeric of Ordinary and Partial Equations. Oxford Univ., Oxford, 342p, 1995.

3. G. Adomian, Solving Frontier Problems of Physics the Decomposition Method, Kluer academic Publisher, Boston, 1994.

4. G. Yüksel, M. Gülsu, M. Sezer, Chebyshev polynomial solutions of aclass of secondorder nonlinear ordinary differential equations, Journal of Advanced Research in Scientific Computing 3(4), 11 - 24 Online ISSN: 1943 -2364, 2011.

5. M. A. El-Tawil, A. A. Bahnasawi, A. Abdel - Naby, Solving Riccati differential equation using Adomians decomposition method, Applied Mathematics and Computation 157, 503 - 514, doi: 10.1016/j.amc.2003.08.049, 2004.

6. V. Hernandez, J. J Ibonez, J. Peinodo, E. Arias, A GMRES-based BDF method for solving Differential Riccati Equations, Applied Mathematics and Computation doi: 10.1016/j. amc.2007.06.021, 2007.

7. Ş. Nas, S. Yalçınbaş and M. Sezer, A Taylor polynomial approach for solving highorder linear Fredholm integro-differential equations, International Journal of Mathematical Education in Science and Technology 31(2), 213 - 225, 2000.

8. S. Yalçınbaş and M. Sezer, The approximate solution of high-order linear VolterraFredholm integrodifferential equations in terms of Taylor polynomials, Applied Mathematics and Computation 112, 291 - 308, 2000.

9. S. Yalçınbaş, Taylor polynomial solutions of nonlinear Volterra-Fredholm integral equations, Applied Mathematics and Computation 127, 195 - 206, 2002.

10. S. Yalçınbaş, K. Erdem, Approximate solution of nonlinear Volterra integral equation systems, International Journal of Modern Physics B 24, 32, 6235 - 6258, 2010.

11. S. Yalçınbaş, M. Sezer, A Taylor collocation method for the approximate solution of general linear Fredholm-Volterra integro-difference equations with mixed argument, Applied Mathematics and Computation 175 (1), 675-690, 2006.

12. K. Erdem, S. Yalçınbaş, Bernoulli Polynomial Approach to High-Order Linear Differential-Difference Equations, AIP Conference Proceedings 1479, 360-364, 2012.

13. S. Yalçınbaş, M. Aynigül, T. Akkaya, Legendre series solutions of Fredholm integral equations, Mathematical and Computational Applications 15(3), 371-381, 2010. 